\title{
Exercise PET: More insight or more complex?
}

\author{
K. Lance Gould, MD, and Nils P. Johnson, MD, MS \\ Division of Cardiology, Department of Medicine, Weatherhead PET Center for Preventing and \\ Reversing Atherosclerosis, University of Texas Medical School at Houston and Memorial \\ Hermann Hospital, Houston, TX
}

Received Feb 15, 2015; accepted Feb 15, 2015

doi: 10.1007/s12350-015-0099-2

\section{See related article, pp. 1273-1280}

In this issue of the journal, Aggarwal et $\mathrm{al}^{1}$ report on 265 patients undergoing diagnostic treadmill stress myocardial perfusion imaging by PET using N-13 ammonia and rotating rod attenuation correction. 194 patients were obese (74\%) with average treadmill capacity significantly less than non-obese patients as reflected by significantly lower exercise duration, functional aerobic capacity, METs achieved, and pressurerate product. Coronary angiograms were done in 43 (16\% of 265) patients of whom 36 (14\% of 265) had significant CAD by visual assessment. Diagnostic sensitivity was $86 \%$ and specificity $74 \%$, with no difference between obese and non-obese patients. The authors conclude that treadmill stress perfusion PET is "feasible

Funding K. Lance Gould received internal funding from the Weatherhead PET Center for Preventing and Reversing Atherosclerosis. He is also the $510(\mathrm{k})$ applicant for cfrQuant approved by the FDA. He has arranged that all his royalties permanently go to a University of Texas scholarship fund. UT has a commercial non-exclusive agreement with Positron Corporation to distribute and market cfrQuant in exchange for royalties. However, he retains the ability to distribute cost-free versions to selected collaborators for research. Nils P. Johnson received internal funding from the Weatherhead PET Center for Preventing and Reversing Atherosclerosis. His institution has received significant institutional research support from Volcano Corporation (for study NCT02328820) and St Jude Medical (for study NCT02184117), companies that make intracoronary pressure and flow sensors.

Reprint requests: K. Lance Gould, MD, Division of Cardiology, Department of Medicine, Weatherhead PET Center for Preventing and Reversing Atherosclerosis, University of Texas Medical School at Houston and Memorial Hermann Hospital, 6431 Fannin St., Room MSB 4.256, Houston, TX 77030; k.lance.gould@uth.tmc.edu

J Nucl Cardiol 2015;22:1281-4.

$1071-3581 / \$ 34.00$

Copyright (c) 2015 American Society of Nuclear Cardiology. and useful clinically with higher rate of good image quality, greater spatial, temporal, and contrast resolution, robust attenuation correction, high-count statistics, and less hepatobiliary tracer uptake of PET compared to SPECT.',

The authors deserve credit for a large series of patients using a treadmill exercise PET perfusion imaging protocol. Due to the short half-life of cyclotron-produced N-13 ammonia, simply timing and coordinating the exercise stress with producing $\mathrm{N}-13$ ammonia for injection at peak exercise and moving patients into the scanner gantry for adequate count images are a singular achievement. The effort to get good relative perfusion images is worthwhile given the attenuation artifacts of SPECT and its lack of management impact in the literature. ${ }^{2,3}$

However, every solid study advances our understanding beyond its conclusions by its instructive flaws or lessons of omission. While a compliment to an experienced group for a difficult study showing feasibility of exercise PET, their report raises significant fundamental physiologic, technical, and management issues about stress perfusion imaging that this paper and the field of nuclear cardiology have not addressed adequately.

\section{WHAT GOOD IS MYOCARDIAL PERFUSION IMAGING?}

In essence, myocardial stress perfusion imaging may be used in two ways. The first and more common use provides qualitative images for diagnosis and prognosis like SPECT, and PET in this paper, including visual scoring from a 17 -segment map. The second but less common use provides objective physiologic severity of focal and diffuse CAD utilizing the full capability of quantitative PET for absolute perfusion, CFR, and automated size and severity of relative and absolute perfusion abnormalities to guide revascularization and management. ${ }^{4,5}$ 
Perfusion imaging by SPECT, and by PET in this paper, traditionally inform diagnosis or prognosis of $\mathrm{CAD}$ without adequate quantification for management and revascularization decisions that are therefore made by coronary angiogram and, less commonly, by pressure-derived fractional flow reserve (FFR). Randomized trials incorporating non-invasive imaging to guide management have not reduced hard endpoints of death and myocardial infarction.

Rather, the literature shows no reduction in adverse outcomes when management is guided by perfusion imaging or by any non-invasive test. ${ }^{2,3}$ The perfusion imaging components of randomized trials such as STICH $^{6}$ and COURAGE ${ }^{7}$ also failed to show reduced hard endpoints by perfusion-guided intervention. Therefore, evidenced-based support does not exist for guiding management by perfusion imaging or any other noninvasive testing.

\section{QUALITATIVE DIAGNOSIS VS QUANTITATIVE PHYSIOLOGIC SEVERITY OF CAD FOR MANAGEMENT}

The randomized FAME $2 \operatorname{trial}^{8}$ demonstrated that quantitative physiologic stenosis severity as measured by FFR is the optimal guide to revascularization; alternatively, risk factors and coronary calcium suffice for guiding medical management. However, the FFR threshold of 0.8 in FAME 2 was not associated with reduced MI or mortality between the medical and revascularization arms due to an elevated 9-to-1 ratio of peri-procedure events within the first week. ${ }^{8}$ Recent meta-analysis reveals a continuous relationship between FFR and the risk of clinical events, ${ }^{9}$ with a rising benefit well below the 0.8 threshold used in FAME 2 .

Additional literature documents that the risk of adverse events increases dramatically with progressively severe focal and diffuse CAD. ${ }^{4,5,10-13}$ Quantitative PET for CFR shows a high risk of MACE for global CFR of 1.5 or less indicating severe diffuse $\mathrm{CAD}^{10}$ that may be moderated by coronary bypass surgery, ${ }^{11}$ whereas revascularization for less severe disease provided no benefit. Thus, quantitative severity of focal and diffuse $\mathrm{CAD}$ is necessary to guide management and revascularization for reduced hard endpoints, not just diagnosis and prognosis.

Importantly, FFR yields only a relative CRF based on the hyperemic pressure gradient across a stenosis. It was originally validated by comparison to quantitative PET $^{14}$ that measured absolute perfusion and CFR. In addition to automated maps of size and severity of stress flow and CFR, automated objective quantitative relative stress perfusion maps are extensively documented. ${ }^{4,5}$

\section{CRITICAL BALANCE BETWEEN QUANTITATIVE SEVERITY OF STENOSIS AND DIFFUSE CAD}

Diffuse CAD quantified by reduced global CFR carries high risk of adverse events. ${ }^{4,5,10-13}$ Since significant stenosis is usually associated with diffuse disease, the residual diffuse disease may contravene the potential benefit by PCI of stenosis, as also evidenced by a significant risk of residual FFR after successful PCI. ${ }^{9}$ For PCI to reduce MI or mortality, a stenosis has to be severe enough to pose a greater cumulative risk than that from residual diffuse disease plus the revascularization procedure itself. For example, in FAME $2,{ }^{8}$ the increased hazard ratio of 9-to-1 (95\% confidence interval 1.13-72.0) for death or MI compared to medical therapy within 7 days of PCI balanced out any reduction of MACE in the PCI group after the first week.

Quantifying the anatomic severity of cumulative, diffuse disease in terms or units directly comparable to focal stenosis is essential for assessing their relative contribution to risk or symptoms. Global and regional CFR and absolute perfusion provide comparable measures of diffuse disease and of focal stenosis for their severity, size as percent of LV in specific arterial distribution, as well as the global burden of diffuse disease. ${ }^{4,5}$ These quantitative measures of size, severity, distribution, and global diffuse disease then identify sufficiently high risk, for which revascularization is likely to reduce MACE. Moreover, they also individualize for each patient between PCI for single stenosis or bypass surgery for diffuse or multivessel disease.

\section{WHAT IS GAINED AND LOST BY EXERCISE PERFUSION PET?}

As the authors state, treadmill exercise precluded measuring the radionuclide arterial input function with dynamic myocardial imaging to determine quantitative perfusion and CFR. Therefore, the greatest strength of PET-objective quantification of absolute perfusion and CFR - is lost with treadmill stress. Therefore, one might reasonably ask what was gained by exercise stress over pharmacologic stress?

While PET images are likely superior to SPECT images due to attenuation correction, the sensitivity of $86 \%$ and specificity of $74 \%$ are not dramatically better than typically reported for SPECT. Since the patients did not have both PET and SPECT for direct comparison, the advantage of PET over SPECT is implied but not proven in this paper but has been in other reports. ${ }^{15}$ PET images were assessed by qualitative visual scores rather 
than automated objective size and severity of relative uptake as reported in other studies. ${ }^{4,5}$ Finally, visually assessed percent diameter stenosis on angiogram is a notoriously inadequate reference "gold standard" of severity for stenosis or diffuse CAD. ${ }^{4,5}$

\section{TREADMILL EXERCISE VS PHARMACOLOGIC VASODILATION FOR STRESS PERFUSION IMAGING}

Additionally, the diagnostic sensitivity of $86 \%$ and specificity of $74 \%$ with treadmill exercise PET in this study remains inferior to over $90 \%$ sensitivity and specificity typically reported for PET with pharmacologic stress. These results suggest that exercise PET is less accurate than pharmacologic vasodilator stress PET. However, without direct comparison of both stressors in the same patient, any differences remain unproven.

Other studies have compared exercise stress with pharmacologic vasodilator stress by both PET and SPECT perfusion imaging. ${ }^{16-18}$ Exercise stress may cause more severe perfusion defects than pharmacologic stress due to sympathetically driven vasoconstriction of subcritical stenosis as demonstrated by exercise angiogram. ${ }^{19}$ However, perfusion abnormalities with pharmacologic stress indicate the pure structural severity of stenosis for revascularization decisions without exercise-induced vasoconstriction ${ }^{18}$ that is optimally treated medically rather than by procedures.

Obesity limits exercise capacity just like musculoskeletal impairment, demonstrated in this study where the obese patients failed to achieve the same duration of exercise, functional aerobic capacity, or metabolic equivalents (METs) as the non-obese group despite less comorbidity (the non-obese group had more known $\mathrm{CAD}$ and revascularization procedures with comparable prevalence of angina, SSS scores, and visually graded "significant" stenosis at angiography). Therefore, the purported value of exercise testing in the obese may be contravened by an inability to achieve adequate exercise levels due to obesity rather than CAD.

\section{TECHNICAL PET IMAGING LESSONS}

The authors report that their PET scanner did not have software for correcting emission-transmission misregistration, well documented as a significant problem for both rotating rod and CT attenuation correction. ${ }^{20,21}$ Transmission data acquired by rotating rod as in this study is associated with significant attenuation-emission misregistration in $20 \%$ of cases when objectively systematically measured. ${ }^{20}$ This misregistration occurs when the heart and diaphragm change position in the thorax during breathing and when abdominal contents slowly shift upward while lying down in the gantry. ${ }^{20,21}$ Such anatomic changes within the body profoundly alter the attenuation data despite no external change in body position compared to laser markers.

The transition from hard treadmill exercise with rapid deep breathing and tachycardia to lying in the scanner with changing respiratory rate, slowing heart rate, and slow repositioning of abdominal and thoracic contents combine to produce internal changes in attenuation anatomy even with no change in external body position. Therefore, the absence of checking and correcting attenuation-emission misregistration certainly contributed to the low specificity of $74 \%$ reported in this manuscript, substantially greater than reported for other PET studies.

While not stated, the rotating rod PET scanner likely operated in 2D mode. In that case, the $300 \mathrm{M}$ counts in the attenuation image over 10 minutes requires an attenuation acquisition of $500,000 \mathrm{cts} / \mathrm{s}$ that is much higher than reported previously for rotating rod 2D scanners. ${ }^{20}$ Such high count rates for a rotating rod source would require a very high activity rod or $3 \mathrm{D}$ acquisition. Separately from treadmill exercise's precluding quantitative perfusion, as the authors acknowledge, a 3D acquisition would likely also preclude quantitative perfusion measurements due to scanner saturation on arterial first pass after a full dose radionuclide bolus.

\section{WHAT IS NEEDED IN NUCLEAR CARDIOLOGY?}

No randomized non-invasive imaging trial to guide management has reduced MI or death. Similarly, no randomized trial of PET-guided management has proven a downstream reduction in unnecessary procedures, MI, death, or hospitalizations. However, non-randomized studies do show reduced hard endpoints as pilot data including thresholds of severity likely necessary proving reduced MI and mortality in a future randomized trial. ${ }^{4,5,11,22,23}$

By measuring size as percent of LV with quantitative severity by absolute stress flow and CFR, vasodilator stress perfusion PET can provide more comprehensive physiologic quantification of CAD than FFR lacking size-severity distribution of focal or diffuse CAD. While FFR was originally validated by comparison to the "gold standard" of PET relative CFR, ${ }^{14}$ current science supports an evolving "gold standard", toward clinical outcomes, not imaging endpoints.

For nuclear cardiology to move beyond the limitations of unproven SPECT and to thrive as a scientifically based contribution to patient betterment, the potential power of quantitative vasodilator stress PET needs a 
randomized trial to prove its worth (or not) for guiding CAD management by reducing $\mathrm{MI}$ or death, and preventing unnecessary procedures. Such a trial, positive or negative, would profoundly alter cardiovascular medicine for optimal patient care and cost benefit.

\section{References}

1. Aggarwal NR, Drozdova A, Askew III JW, Kemp BJ, Chareonthaitawee P. Feasibility and diagnostic accuracy of exercise treadmill nitrogen-13 ammonia PET myocardial perfusion imaging of obese patients. J Nucl Cardiol 2015. doi:10.1007/s12350015-0073-z.

2. Hachamovitch R, Nutter B, Hlatky MA, Shaw LJ, Ridner ML, Dorbala $\mathrm{S}$, et al. Patient management after noninvasive cardiac imaging. Results from SPARC. J Am Coll Cardiol 2012;59:46274.

3. Patel MR, Dai D, Hernandez AF, Douglas PS, Messenger J, Garratt KN, et al. Prevalence and predictors of non-obstructive coronary artery disease identified with coronary angiography in contemporary clinical practice. Am Heart J 2014;167:846-52.

4. Gould KL, Johnson NP, Bateman TM, Beanlands RS, Bengel FM, Bober R, et al. Anatomic versus physiologic assessment of coronary artery disease: Role of CFR, FFR, and PET imaging in revascularization decision-making. J Am Coll Cardiol 2013;62: 1639-53.

5. Johnson NP, Gould KL. Integrating noninvasive absolute flow, coronary flow reserve, and ischemic thresholds into a comprehensive map of physiologic severity. J Am Coll Cardiol 2012;5:430-40.

6. Panza JA, Holly TA, Asch FM, She L, Pellikka PA, Velazquez EJ, et al. Inducible myocardial ischemia and outcomes in patients with coronary artery disease and left ventricular dysfunction-Substudy of STICH with reversible ischemia by SPECT. J Am Coll Cardiol 2013;61:1860-70.

7. Boden WE, O'Rourke RA, Teo KK, Hartigan PM, Maron DJ, Kostuk WJ, et al. Optimal medical therapy with or without PCI for stable coronary disease. N Engl J Med 2007;356:1503-16.

8. De Bruyne B, Fearon WF, Pijls NH, Barbato E, Tonino P, Piroth $\mathrm{Z}$, et al. Fractional flow reserve-guided PCI for stable coronary artery disease. N Engl J Med 2014;371:1208-17.

9. Johnson NP, Tóth GG, Lai D, Zhu H, Açar G, Agostoni P, et al. Prognostic value of fractional flow reserve (FFR): Linking physiologic severity to clinical outcomes. J Am Coll Cardiol 2014;64:1641-54.

10. Murthy VL, Naya M, Foster CR, Hainer J, Gaber M, Di Carli G, et al. Improved cardiac risk assessment with noninvasive measures of coronary flow reserve. Circulation 2011;124:2215-24.

11. Taqueti VR, Hachamovitch R, Murthy VL, Naya M, Foster CR, Hainer $\mathrm{J}$, et al. Global coronary flow reserve associates with adverse cardiovascular events independently of luminal angiographic severity, and modifies the effect of early revascularization. Circulation 2015;131:19-27. doi:10.1161/CIRCULATIONAHA. 114.011939.

12. Gould KL, Johnson NP. Physiologic severity of diffuse CADHidden high risk. Circulation 2015;131:4-6. doi:10.1161/ CIRCULATIONAHA.114.013815.

13. Gould KL, Johnson NP. Physiologic stenosis severity, binary thinking, revascularization, and hidden reality. Circ CV Imaging 2015. doi:10.1161/CIRCIMAGING.114.002970.

14. De Bruyne B, Baudhuin T, Melin JA, Pijls NH, Sys SU, Bol A, et al. Coronary flow reserve calculated from pressure measurements in humans. Validation with positron emission tomography. Circulation 1994;89:1013-22.

15. Bateman TM, Heller GV, McGhie AI, Friedman JD, Case JA, Bryngelson JR, et al. Diagnostic accuracy of rest/stress ECG-gated Rb-82 myocardial perfusion PET: Comparison with ECG-gated Tc-99m sestamibi SPECT. J Nucl Cardiol 2006;13:24-33.

16. Chow BJW, Beanlands RS, Lee A, DaSilva JN, deKemp RA, Alkahtani A, et al. Treadmill exercise produces larger perfusion defects than dipyridamole stress n-13 ammonia positron emission tomography. J Am Coll Cardiol 2006;47:411-6.

17. Verna E, Ceriani L, Provasoli S, Scotti S, Ghiringhelli S. Larger perfusion defects with exercise compared with dipyridamole SPECT (exercise-dipyridamole mismatch) may reflect differences in epicardial and microvascular coronary dysfunction: When the stressor matters. J Nucl Cardiol 2007;14:818-26.

18. Schindler TH, Schelbert HH. "Mismatch"' in regional myocardial perfusion defects during exercise and pharmacologic vasodilation: Anoninvasive marker of epicardial vasomotor dysfunction? J Nucl Cardiol 2007; 14:769-74.

19. Gordon JB, Ganz P, Nabel EG, Fish RD, Zebede J, Mudge GH, et al. Atherosclerosis influences the vasomotor response of the epicardial coronary arteries to exercise. J Clin Invest 1989;83: 1946-52.

20. Loghin C, Sdringola S, Gould KL. Common artifacts in PET myocardial perfusion images due to attenuation-emission misregistration: Clinical significance, causes and solutions in 1177 patients. J Nucl Med 2004;45:1029-39.

21. Gould KL, Pan T, Loghin C, Johnson N, Guha A, Sdringola S. Frequent diagnostic errors in cardiac PET-CT due to misregistration Of CT attenuation and emission PET images: A definitive analysis of causes, consequences and corrections. J Nucl Med 2007;48:1112-21

22. Sdringola S, Nakagawa K, Nakagawa Y, Yusuf W, Mullani N, Haynie $\mathrm{M}$, et al. Combined intense lifestyle and pharmacologic lipid treatment further reduce coronary events and myocardial perfusion abnormalities compared to usual care cholesterol lowering drugs in coronary artery disease. J Am Coll Cardiol 2003;41:262-72.

23. Sdringola S, Boccalandro F, Loghin C, Gould KL. Mechanisms of progression and regression of coronary artery disease by PET related to treatment intensity and clinical events at long-term followup. J Nucl Med 2006;47:59-67. 